\title{
Spectroscopic and photometric analysis of the early-type spectroscopic binary HD 161853 in the centre of an $\mathrm{H}$ II region (Research Note)
}

\author{
R. Gamen ${ }^{1}$, C. Putkuri ${ }^{1, \star}$, N. I. Morrell ${ }^{2}$, R. H. Barbá ${ }^{3}$, J. I. Arias ${ }^{3}$, J. Maíz Apellániz ${ }^{4}$, \\ N. R. Walborn ${ }^{5}$, A. Sota $^{6}$, and E. J. Alfaro 6

\begin{abstract}
${ }^{1}$ Instituto de Astrofísica de La Plata (CONICET La Plata - UNLP), Facultad de Ciencias Astronómicas y Geofísicas, Universidad Nacional de La Plata, Paseo del Bosque s/n, B1900FWA, La Plata, Argentina e-mail: rgamen@fcaglp.unlp.edu.ar

2 Las Campanas Observatory, Carnegie Observatories, Casilla 601, La Serena, Chile

3 Departamento de Física y Astronomía, Universidad de La Serena, Cisternas 1200 Norte, La Serena, Chile

4 Centro de Astrobiología (CSIC-INTA), ESAC Campus, PO Box 78, 28691 Villanueva de la Cañada, Madrid, Spain

5 Space Telescope Science Institute ${ }^{\star \star}, 3700$ San Martin Drive, Baltimore, MD 21218, USA

${ }^{6}$ Instituto de Astrofísica de Andalucía-CSIC, Glorieta de la Astronomía s/n, 18008 Granada, Spain
\end{abstract}

Received 19 December 2014 / Accepted 5 October 2015

\section{ABSTRACT}

\begin{abstract}
Aims. We study the O-type star HD 161853, which has been noted as a probable double-lined spectroscopic binary system. Methods. We secured high-resolution spectra of HD 161853 during the past nine years. We separated the two components in the system and measured their respective radial velocities for the first time.

Results. We confirm that HD 161853 is an $\sim 1$ Ma old binary system consisting of an O8 V star $\left(M_{\mathrm{A}, \mathrm{RV}} \geq 22 M_{\odot}\right)$ and a B1-3 V star $\left(M_{\mathrm{B}, \mathrm{RV}} \geq 7.2 M_{\odot}\right)$ at about $1.3 \mathrm{kpc}$. From the radial velocity curve, we measure an orbital period $P=2.66765 \pm 0.00001 \mathrm{~d}$ and an eccentricity $e=0.121 \pm 0.007$. Its $V$-band light curve is constant within 0.014 mag and does not display eclipses, from which we impose a maximum orbital inclination $i=54 \mathrm{deg}$. HD 161853 is probably associated with an $\mathrm{H}$ II region and a poorly investigated very young open cluster. In addition, we detect a compact emission region at 50 arcsec to HD 161853 in $22 \mu \mathrm{m}$-WISE and $24 \mu \mathrm{m}-S p i t z e r$ images, which may be identified as a dust wave piled up by the radiation pressure of the massive binary system.
\end{abstract}

Key words. binaries: spectroscopic - stars: early-type - stars: individual: HD 161853

\section{Introduction}

HD 161853 (=CPD -31 4999; RA (J2000) = 17:49:16.6, $\operatorname{Dec}(\mathrm{J} 2000)=-31: 15: 18 ; l=358.4248, b=-1.8767 ; V_{J}=$ $7.9 \mathrm{mag}$ ) is an O-type star that has been considered in the literature as either a massive or a post-AGB star. It was first included in the Henry Draper Catalogue (Cannon \& Pickering 1924) and classified as type B3. Subsequently, Crampton (1971, 1972), who also reported radial velocity variations, and Walborn (1973) classified it as O7.5 and O8 V((n)), respectively.

New observations of HD 161853 in the mid- and far-infrared led to confusion about its true nature. For instance, Ratag et al. (1990) proposed it to be a planetary nebula candidate because its radio emission at $4.8 \mathrm{GHz}$ and IRAS colours were typical of such objects. Furthermore, Parthasarathy (1993) considered it a post-AGB star rapidly evolving toward a young planetary nebula nucleus. Although Szczerba et al. (2007) disqualified it as a post-AGB star because of its high effective temperature of more than $35000 \mathrm{~K}$, they did not clarify its actual nature, and more works continued considering HD 161853 as a low-tointermediate-mass star (e.g. Cerrigone et al. 2011).

* While fellow of the Consejo Interuniversitario Nacional of Argentina.

$\star \star$ Operated by AURA, Inc., under NASA contract NAS5-2655.
HD 161853 is located at the centre of an H II region associated with a CO molecular cloud (Blitz et al. 1982) at a kinematic distance of $1.5 \pm 0.2 \mathrm{kpc}$ (Hou \& Han 2014). There has also been some confusion because the region was catalogued by Sharpless (1953, 1959) with different running numbers (Sh 1-17 and Sh 2-15). In addition, the $\mathrm{H}$ II region is not unequivocally identified in the SIMBAD database and has many unrelated entries, such as RCW 134 (Rodgers et al. 1960), W 25 (Westerhout 1958), and G358.464-01.897 (Anderson et al. 2014). The nebula was also identified at $\mathrm{H} \alpha$ by Gum (1955) as Gum 69 (in the SIMBAD database, Gum 69 is used as an alternative identifier of the star HD 161853).

In the field of HD 161853 there are also other objects, suggesting that the area is a young region that deserves further studies. For example, there are several reported young star candidates (Robitaille et al. 2008), and the open cluster Dutra-Bica 51 (Dutra \& Bica 2000). Dias et al. (2002) determined a distance of $1.3 \mathrm{kpc}$, an apparent diameter of $2.2 \mathrm{arcmin}$, and an age of only $1 \mathrm{Ma}$ for the cluster. However, de Wit et al. (2004) did not find a stellar overdensity in the field that could be related to a cluster. A supernova remnant, G358.4-01.9, with dimensions $40 \times 36$ arcmin, whose centre is 1.5 arcmin south of HD 161853, was reported by Reich et al. (1988). However, Gray (1994) ruled out the existence of such a remnant because the region presents a thermal spectral distribution. Finally, 
Table 1. Details of the spectroscopic data for HD 161853.

\begin{tabular}{llccc}
\hline \hline Instr. Config. & Observatory & $R$ & $\begin{array}{c}\Delta \lambda \\
{[\AA]}\end{array}$ & $N$ \\
\hline Échelle, 2.5-m & LCO & 40000 & $3450-9850$ & 13 \\
REOSC, 2.15-m & CASLEO & 15000 & $3600-6100$ & 26 \\
FEROS, 2.2-m & La Silla & 46000 & $3570-9210$ & 4 \\
\hline
\end{tabular}

there is a faint ROSAT source at the position of HD 161853 , 1RXS J174916.5-311509 (Voges et al. 2000), also detected by Chandra (CXOGBS J174916.6-311518; Jonker et al. 2014; Albacete-Colombo, in prep.).

Multiplicity of HD 161853 has been suspected since the publications of Crampton $(1971,1972)$. More recently, Mello et al. (2012) noted double He I absorption lines that were related to a binary nature of the source, and Sota et al. (2014) identified them as coming from an $\mathrm{O} 8 \mathrm{~V}(\mathrm{n}) \mathrm{z}$ and B-type components, but they did not compute any orbit determination.

In this paper, we present the spectral classifications of the two components of HD 161853 and their respective radial velocity orbits. We also analyse the available images of the field and use the ASAS $V$-band data to constrain the orbital inclination. Finally, we discuss the actual nature of HD 161853. Preliminary results of this work were shown by Putkuri et al. (2014).

\section{Observations}

This work is based on observations obtained within the intensive spectroscopic campaign named the OWN Survey (Barbá et al. 2010). We employed the REOSC Cassegrain spectrograph in cross-dispersed mode at the Complejo Astronómico El Leoncito (CASLEO) in Argentina, along with FEROS at the $2.2 \mathrm{~m}$ telescope of ESO/La Silla, and the échelle spectrograph at the $2.5 \mathrm{~m}$ du Pont telescope of Las Campanas Observatory (LCO), in Chile. Observations were secured between 2006 May and 2013 August. The instrumental configuration, observatory, spectral resolving power, wavelength coverage, and the number of obtained spectra $(N)$ are summarised in Table 1.

At CASLEO and LCO, we obtained a wavelength calibration lamp (Th-Ar) exposure immediately before or after each target integration, at the same telescope position. The spectra were processed and calibrated using standard IRAF $^{1}$ routines. For FEROS, we applied the standard reduction pipeline provided by ESO, which uses comparison lamp exposures obtained at the beginning and end of each observing night.

\section{Results and discussion}

\subsection{Radial velocities}

Some of our spectra of HD 161853 clearly show two components, as noted by Mello et al. (2012) and Sota et al. (2014). Thus, we employed the method for separating composite spectra as explained in González \& Levato (2006). After a few iterations, we obtained the individual spectra of HD $161853 \mathrm{~A}$ and B and radial velocities for the 43 observed epochs. Some Balmer lines showed issues due to incorrect normalisation of the échelle orders containing broad lines.

\footnotetext{
IRAF is distributed by the National Optical Astronomy Observatories, which are operated by the Association of Universities for Research in Astronomy, Inc., under cooperative agreement with the National Science Foundation.
}

Table 2. Radial velocities of the two components in HD 161853.

\begin{tabular}{cccccc}
\hline \hline HJD & $\mathrm{RV}_{\mathrm{A}}$ & $\mathrm{RV}_{\mathrm{B}}$ & Weight & $\begin{array}{c}\text { Phase } \\
\text { Obs }\end{array}$ \\
\hline 2453867.879 & 25 & -36 & 0.5 & 0.07 & $\mathrm{CAS}$ \\
2453875.893 & 33 & -84 & 0.5 & 0.08 & LCO \\
2454222.835 & 63 & -192 & 1.0 & 0.13 & CAS \\
2454246.821 & 58 & -174 & 1.0 & 0.12 & ESO \\
2454251.822 & -17 & 106 & 0.5 & 1.00 & CAS \\
2454252.804 & 84 & -236 & 1.0 & 0.36 & CAS \\
2454253.667 & -52 & 216 & 1.0 & 0.69 & CAS \\
2454257.897 & 94 & -276 & 1.0 & 0.27 & LCO \\
2454258.640 & 5 & -16 & 0.5 & 0.55 & LCO \\
2454258.882 & -45 & 136 & 1.0 & 0.64 & LCO \\
2454286.600 & 32 & $\ldots$ & 1.0 & 0.03 & CAS \\
2454286.753 & 39 & $\ldots$ & 1.0 & 0.09 & CAS \\
2454312.603 & -97 & 296 & 1.0 & 0.78 & CAS \\
2454316.490 & 95 & -272 & 1.0 & 0.24 & CAS \\
2454316.611 & 98 & -271 & 1.0 & 0.28 & CAS \\
2454339.469 & -95 & $\ldots$ & 1.0 & 0.85 & CAS \\
2454339.584 & -80 & 255 & 1.0 & 0.90 & CAS \\
2454340.506 & 99 & -267 & 1.0 & 0.24 & CAS \\
2454340.608 & 104 & -268 & 1.0 & 0.28 & CAS \\
2454608.891 & -85 & 315 & 1.0 & 0.85 & CAS \\
2454643.765 & -70 & 252 & 1.0 & 0.92 & CAS \\
2454659.757 & -74 & 248 & 1.0 & 0.92 & CAS \\
2454953.769 & 75 & -208 & 1.0 & 0.13 & ESO \\
2454955.765 & -82 & 256 & 1.0 & 0.88 & ESO \\
2454956.780 & 95 & -272 & 1.0 & 0.26 & ESO \\
2454960.790 & -90 & 280 & 1.0 & 0.76 & LCO \\
2454961.818 & 68 & -218 & 1.0 & 0.15 & LCO \\
2454961.830 & 71 & -226 & 1.0 & 0.15 & LCO \\
2454963.715 & -100 & 328 & 1.0 & 0.86 & LCO \\
2454963.723 & -95 & 308 & 1.0 & 0.86 & LCO \\
2454963.921 & -69 & 247 & 1.0 & 0.93 & LCO \\
2454964.662 & 92 & -261 & 1.0 & 0.21 & LCO \\
2454964.922 & 95 & -265 & 1.0 & 0.31 & LCO \\
2454984.868 & -90 & 310 & 1.0 & 0.79 & CAS \\
2455846.507 & -88 & 283 & 1.0 & 0.78 & LCO \\
2456521.470 & -89 & 250 & 1.0 & 0.80 & CAS \\
2456521.678 & -82 & 246 & 1.0 & 0.88 & CAS \\
2456522.473 & 86 & -219 & 1.0 & 0.18 & CAS \\
2456522.659 & 102 & -255 & 1.0 & 0.25 & CAS \\
2456523.605 & -29 & 67 & 1.0 & 0.60 & CAS \\
2456523.707 & -41 & 127 & 1.0 & 0.64 & CAS \\
2456524.524 & -67 & 193 & 1.0 & 0.95 & CAS \\
2456524.687 & -31 & 71 & 1.0 & 0.01 & CAS \\
\hline & & & & &
\end{tabular}

We performed the cross-correlation by using the FXCOR task of IRAF. The wavelength regions considered were 5008-5024 and 5864-5888 $\AA$, which include the He I $\lambda 5015$ and $\lambda 5875$ absorption lines present in both components. FXCOR provides errors, but as they are smaller than the expected instrumental errors, we adopted a conservative error of $2.5 \mathrm{~km} \mathrm{~s}^{-1}$ for all measurements. Moreover, the interstellar Na I $\lambda \lambda 5890,5896 \AA$ measured in the spectra resulted in a mean of $-10 \mathrm{~km} \mathrm{~s}^{-1}$ with a maximum difference of $2.5 \mathrm{~km} \mathrm{~s}^{-1}$ among them, and a standard deviation of $1 \mathrm{~km} \mathrm{~s}^{-1}$. We did not find any systematic differences among measurements performed with the three spectrographs used in this work. The heliocentric Julian days (HJD) and radial velocities (RV) of the two components are shown in Table 2.

\subsection{Separated spectra}

We independently analysed and classified the separated spectra following Sota et al. (2011) for the O-type primary, and 


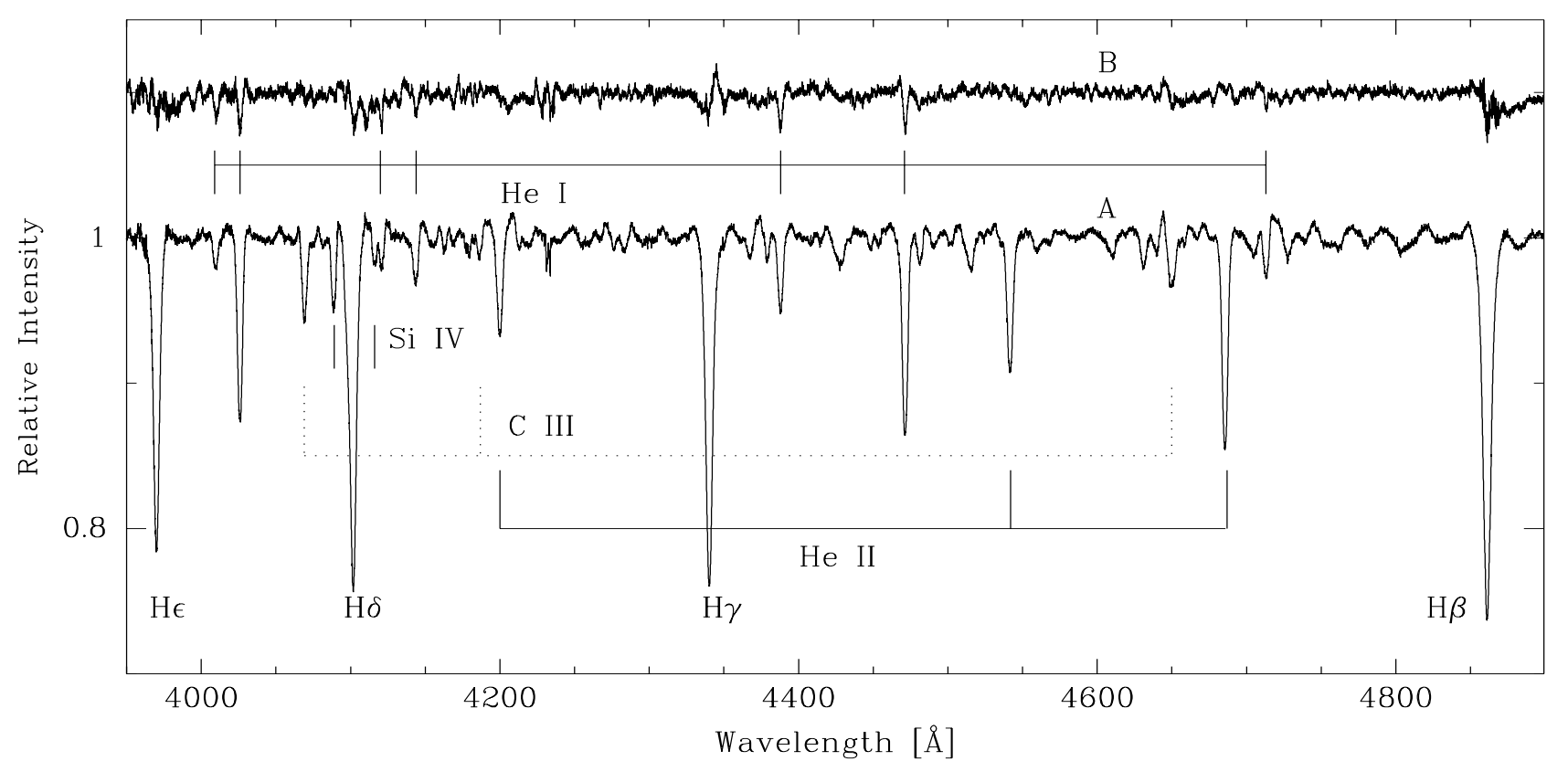

Fig. 1. Separated optical spectra of HD 161853 A and B. The most conspicuous lines are labelled.

following Walborn \& Fitzpatrick (1990) for the B-type secondary component. We inspected the spectra visually using the MGB code (Maíz Apellániz et al. 2012), which allows the user to compare the unknown spectrum with standard stars of each sub-type.

We determined a spectral type $08 \mathrm{Vz}$ for the primary and B1-3 V for the secondary. The $08 \mathrm{Vz}$ classification agrees with Sota et al. (2014). The B1-3 sub-type is only determined from the intensity relations among the faint He I lines. Other lines useful as primary criteria, such as $\mathrm{Mg}$ II $\lambda 4481 \AA$ and Si II $\lambda \lambda 4128,4130 \AA$, are very noisy or affected by residuals in the derived spectrum. The two spectra are shown in Fig. 1.

\subsection{Orbital solution}

The obtained RV measurements of the primary star were used to search for periodicities by means of the Lomb-Scargle algorithm (Scargle 1982). This algorithm is provided on-line as a NASA Exoplanet Archive service (Akeson et al. 2013). A period of 2.66 days was obtained.

The orbital solution of the SB2 was determined with an improved version of the original program for the determination of the orbital elements of spectroscopic binaries (Bertiau \& Grobben 1969), named GBART and developed by F. Bareilles ${ }^{2}$. Some RV values were weighted by 0.5 when the two components did not separate well in the respective spectra (see Table 2). The RVs of both components converged to a slightly eccentric orbit and a relatively low mass ratio. The orbital elements are shown in Table 3 and the RV curves are depicted in Fig. 2.

The non-zero orbital eccentricity implies that the age of the system should be younger than the expected circularisation time, $t_{\text {circ }}$. This value was found to be $t_{\text {circ }} \sim 1$ Ma using Eq. (5.9) in Zahn (1977) and adopting the mass and radius from Martins et al. (2005) and the tidal torque constant $E_{2}=3.5 \times 10^{-6}$ (Zahn 1975). This means that HD 161853 is a young massive system.

\footnotetext{
2 GBART is available at http://www.iar.unlp.edu.ar/ fede/ pub/gbart.
}

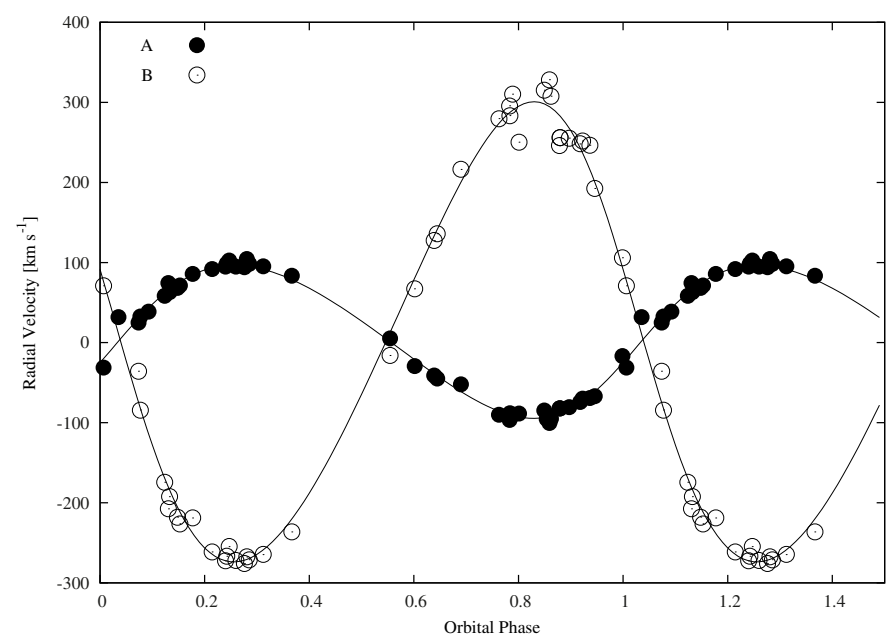

Fig. 2. RV curves of the two components in HD 161853. RV errors are smaller than the size of the symbols. The curves depict the orbital motion calculated from the parameters of Table 3.

Through comparison of the minimum masses in Table 3 with the masses expected from theoretical models shown in Table 4, we estimated possible inclinations of $i=54 \pm 7 \mathrm{deg}$ (using the O star mass) and $i=47 \pm 7 \mathrm{deg}$ (using the B star mass range). The theoretical mass ratio obtained adopting a B3 V sub-type for the secondary star agrees better with the observations than adopting B1 V.

\subsection{Photometric analysis}

The short period of the binary encouraged us to retrieve and analyse the photometry obtained by the All Sky Automated Survey (ASAS, Pojmánski 2002). The data, although near the saturation limit, appear to be almost constant between 2001 and 2009. Statistics performed over 813 values (six-pixel aperture photometry with quality labelled as A) gave a mean of $V=7.956 \mathrm{mag}$, a standard deviation of $0.014 \mathrm{mag}$, and a range of $0.084 \mathrm{mag}$ between the lowest and highest values. Thus, the lack of eclipses 


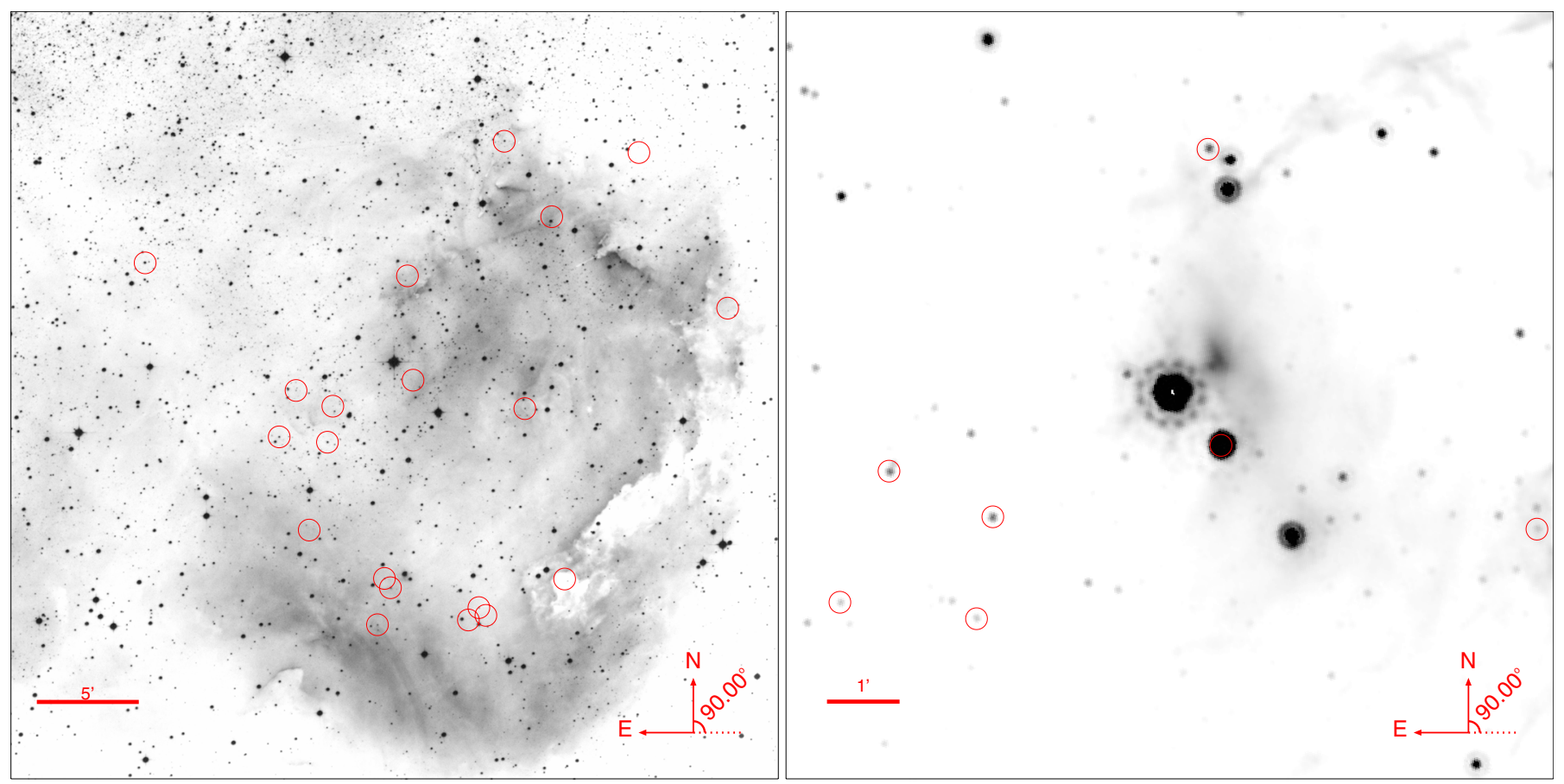

Fig. 3. Images centred on HD 161853. Young stars are indicated with circles. Left panel: $30 \times 30$ arcmin Super COSMOS AAO/UKST H $\alpha$ image (Parker et al. 2005) showing the Sh 2-15 H II region. Right panel: $5.5 \times 5.3$ arcmin Spitzer $24 \mu$ m image showing some structures associated with our star.

Table 3. Orbital parameters of HD 161853 AB.

\begin{tabular}{lc}
\hline \hline Parameter & Value \\
\hline$P[\mathrm{~d}]$ & $2.66765 \pm 0.00001$ \\
$T_{\text {peri }}[\mathrm{HJD}]$ & $2456634.04 \pm 0.02$ \\
$T_{\mathrm{RV} \max }[\mathrm{HJD}]$ & $2456634.73 \pm 0.02$ \\
$V_{0}\left[\mathrm{~km} \mathrm{~s}^{-1}\right]$ & $4.0 \pm 1.0$ \\
$e$ & $0.121 \pm 0.007$ \\
$\omega[\mathrm{deg}]$ & $254 \pm 4$ \\
$q\left(M_{2} / M_{1}\right)$ & $0.332 \pm 0.007$ \\
$K_{1}\left[\mathrm{~km} \mathrm{~s}^{-1}\right]$ & $96 \pm 2$ \\
$K_{2}\left[\mathrm{~km} \mathrm{~s}^{-1}\right]$ & $287 \pm 2$ \\
$M_{1} \sin ^{3} i\left[M_{\odot}\right]$ & $11.4 \pm 0.4$ \\
$M_{2} \sin ^{3} i\left[M_{\odot}\right]$ & $3.8 \pm 0.3$ \\
$a_{1} \sin i\left[R_{\odot}\right]$ & $4.97 \pm 0.08$ \\
$a_{2} \sin i\left[R_{\odot}\right]$ & $14.95 \pm 0.09$ \\
\hline
\end{tabular}

Table 4. Theoretical stellar parameters.

\begin{tabular}{rccc}
\hline \hline Parameter & O8 V & B1 V & B3 V \\
& Martins et al. (2005) & \multicolumn{2}{c}{ Cox (2000) } \\
\hline$M\left[M_{\odot}\right]$ & 21.5 & 14.2 & 7.6 \\
$R\left[R_{\odot}\right]$ & 8 & 6.5 & 4.8 \\
\hline
\end{tabular}

can also be used to constrain the highest orbital inclination. We fitted the spectroscopic and photometric data with a WilsonDevinney model (Wilson \& Devinney 1971) by means of the PHOEBE code (Prša \& Zwitter 2005) and adopting the theoretical stellar parameters shown in Table 4 . We determined a highest orbital inclination of $i=54 \mathrm{deg}$, and hence the masses should be greater than $22 M_{\odot}$ and $7.2 M_{\odot}$ for the $\mathrm{O} 8 \mathrm{~V}$ and B1-3 V stars, respectively. We note the agreement between the value of the inclination angle from photometry and the one from the comparison between the minimum masses and those expected from the theoretical models.

The available multi-band photometry permits analysing the interstellar extinction and determination of the spectrophotometric distance to HD 161853. We applied the CHORIZOS code (Maíz-Apellániz 2004) to the $U B V$ (Sota et al. 2008), Tycho$2 B V$ (Høg et al. 2000), and JHKs (2MASS; Skrutskie et al. 2006) data. We obtained $E(4405-5495)=0.496 \pm 0.011 \mathrm{mag}$, and $A_{\mathrm{V}}=1.82 \pm 0.02 \mathrm{mag}$, which results in a distance modulus $V_{J, 0}-M_{V}=6.242-M_{V}$ mag. Adopting $M_{V}=-4.3$ mag for the O8 Vz star (Walborn 1972; Martins et al. 2005) and that the effect of the flux of the B1-3 V star is smaller than the uncertainty (0.45 mag), we derived a distance of $1.35 \pm 0.25 \mathrm{kpc}$, which is consistent with the kinematic distance to the $\mathrm{CO}$ molecular cloud (Hou \& Han 2014) and to the Dutra-Bica 51 open cluster (Dias et al. 2002). This determination supports the HD 161853 membership in a star-forming region that should be studied with further tailored observations.

The mid-infrared images of WISE $(22 \mu \mathrm{m}$; Wright et al. 2010) and Spitzer/MIPS (24 $\mu \mathrm{m}$; Rieke et al. 2004) reveal a compact emission region 50 arcsec north-west from HD 161853 (see Fig. 3). This feature is not detected in the shorter wavelength bands of Spitzer and WISE. It may be a dust wave, similar to the arc-like cloud near $\sigma$ Ori noted by Caballero et al. (2008). Ochsendorf et al. (2014) explained these regions as the result of surrounding dust piled up by the radiation pressure of the massive star.

\section{Summary}

The O-type star HD 161853 is an SB2 system with massive components. We separated the composite spectrum with a separation method and classified the primary star as $\mathrm{O} 8 \mathrm{Vz}$ and the secondary as B1-3 V. We determined individual RVs for both components and derived the orbital parameters, obtaining a period 
of 2.66765 days and an eccentricity of 0.121 . We also calculated minimum masses of $11.4 M_{\odot}$ and $3.8 M_{\odot}$ for the O- and B-type stars, respectively.

We analysed the photometry of this star available in the ASAS database. The photometry was useful to constrain the orbital inclination to a value lower than $54 \mathrm{deg}$ and hence increasing the minimum masses to 22 and $7.2 M_{\odot}$.

The minimum masses determined for the stars and the eccentricity are the most direct and reliable proof that the O-type component is a massive young star and not a post-AGB object. It is located in a very young region in the centre of an $\mathrm{H}$ II region, consistently with the results of the binary analysis. We have identified a compact emission region 50 arcsec north-west from our star. The mid-infrared flux from this dust wave caused HD 161853 to be erroneously considered as a postAGB star for almost two decades.

Acknowledgements. We thank the referee José A. Caballero for careful reading of our paper and useful suggestions that improved the work. We thank the directors and staffs of CASLEO, LCO, and ESO/La Silla for the use of their facilities and their kind hospitality during the observing runs. CASLEO is operated under agreement between CONICET and the Universities of La Plata, Córdoba and San Juan, Argentina. The Échelle Liège Spectrograph was jointly built by REOSC and Liège Observatory and is on long-term loan from the latter. This research has made use of NASA's Astrophysics Data System, the SIMBAD database operated at CDS, Strasbourg, France, and the Aladin interactive sky atlas developed at CDS.

\section{References}

Akeson, R. L., Chen, X., Ciardi, D., et al. 2013, PASP, 125, 989

Anderson, L. D., Bania, T. M., Balser, D. S., et al. 2014, ApJS, 212, 1

Barbá, R. H., Gamen, R., Arias, J. I., et al. 2010, in Rev. Mex. Astron. y Astrofis. Conf. Ser., 38, 30

Bertiau, F. C., \& Grobben, J. 1969, Ric. Astron., 8, 1

Blitz, L., Fich, M., \& Stark, A. A. 1982, ApJS, 49, 183

Caballero, J. A., Valdivielso, L., Martín, E. L., et al. 2008, A\&A, 491, 515

Cannon, A. J., \& Pickering, E. C. 1924, Henry Draper (HD) catalog and HD extension

Cerrigone, L., Trigilio, C., Umana, G., Buemi, C. S., \& Leto, P. 2011, MNRAS, 412,1137

Crampton, D. 1971, AJ, 76, 260

Crampton, D. 1972, MNRAS, 158, 85

de Wit, W. J., Testi, L., Palla, F., Vanzi, L., \& Zinnecker, H. 2004, A\&A, 425, 937

Dias, W. S., Alessi, B. S., Moitinho, A., \& Lépine, J. R. D. 2002, A\&A, 389, 871
Dutra, C. M., \& Bica, E. 2000, A\&A, 359, L9

González, J. F., \& Levato, H. 2006, A\&A, 448, 283

Gray, A. D. 1994, MNRAS, 270, 835

Gum, C. S. 1955, MmRAS, 67, 155

Høg, E., Fabricius, C., Makarov, V. V., et al. 2000, A\&A, 355, L27

Hou, L. G., \& Han, J. L. 2014, A\&A, 569, A125

Jonker, P. G., Torres, M. A. P., Hynes, R. I., et al. 2014, ApJS, 210, 18

Maíz-Apellániz, J. 2004, PASP, 116, 859

Maíz Apellániz, J., Pellerin, A., Barbá, R. H., et al. 2012, in Proc. Scientific Meeting in Honor of Anthony F. J. Moffat, eds. L. Drissen, C. Rubert, N. StLouis, \& A. F. J. Moffat, ASP Conf. Ser., 465, 484

Martins, F., Schaerer, D., \& Hillier, D. J. 2005, A\&A, 436, 1049

Mello, D. R. C., Daflon, S., Pereira, C. B., \& Hubeny, I. 2012, A\&A, 543, A11

Ochsendorf, B. B., Cox, N. L. J., Krijt, S., et al. 2014, A\&A, 563, A65

Parker, Q. A., Phillipps, S., Pierce, M. J., et al. 2005, MNRAS, 362, 689

Parthasarathy, M. 1993, in Luminous High-Latitude Stars, ed. D. D. Sasselov, ASP Conf. Ser., 45, 173

Pojmánski, G. 2002, Acta Astron., 52, 397

Prša, A., \& Zwitter, T. 2005, ApJ, 628, 426

Putkuri, C., Gamen, R., Morrell, N. I., Barbá, R., \& Arias, J. 2014, in 57 Reunión Anual de la Asociación Argentina de Astronomía, published online at http: //aaa2014.oac. uncor. edu/posters/putkuri.pdf

Ratag, M. A., Pottasch, S. R., Zijlstra, A. A., \& Menzies, J. 1990, A\&A, 233, 181

Reich, W., Fürst, E., Reich, P., \& Junkes, N. 1988, in IAU Colloq. 101: Supernova Remnants and the Interstellar Medium, eds. R. S. Roger, \& T. L. Landecker, 293

Rieke, G. H., Young, E. T., Engelbracht, C. W., et al. 2004, ApJS, 154, 25

Robitaille, T. P., Meade, M. R., Babler, B. L., et al. 2008, AJ, 136, 2413

Rodgers, A. W., Campbell, C. T., \& Whiteoak, J. B. 1960, MNRAS, 121, 103

Scargle, J. D. 1982, ApJ, 263, 835

Sharpless, S. 1953, ApJ, 118, 362

Sharpless, S. 1959, ApJS, 4, 257

Skrutskie, M. F., Cutri, R. M., Stiening, R., et al. 2006, AJ, 131, 1163

Sota, A., Maíz Apellániz, J., Walborn, N. R., \& Shida, R. Y. 2008, in Rev. Mex. Astron. Astrofis. Conf. Ser., 33, 56

Sota, A., Maíz Apellániz, J., Walborn, N. R., et al. 2011, ApJS, 193, 24

Sota, A., Maíz Apellániz, J., Morrell, N. I., et al. 2014, ApJS, 211, 10

Szczerba, R., Siódmiak, N., Stasińska, G., \& Borkowski, J. 2007, A\&A, 469, 799

Voges, W., Aschenbach, B., Boller, T., et al. 2000, IAU Circ., 7432, 1

Walborn, N. R. 1972, AJ, 77, 312

Walborn, N. R. 1973, AJ, 78, 1067

Walborn, N. R., \& Fitzpatrick, E. L. 1990, PASP, 102, 379

Westerhout, G. 1958, Bull. Astron. Inst. Netherlands, 14, 215

Wilson, R. E., \& Devinney, E. J. 1971, ApJ, 166, 605

Wright, E. L., Eisenhardt, P. R. M., Mainzer, A. K., et al. 2010, AJ, 140, 1868

Zahn, J.-P. 1975, A\&A, 41, 329

Zahn, J.-P. 1977, A\&A, 57, 383 